\title{
Sabrina Cotogno \\ Parametrization of the Transverse Momentum Dependent Light-Front Correlator for Gluons
}

Received: 19 January 2017 / Accepted: 31 January 2017 / Published online: 17 February 2017

(C) The Author(s) 2017. This article is published with open access at Springerlink.com

\begin{abstract}
We study the transverse momentum dependent light-front correlator for gluons. At the operator level this is expressed as a matrix element containing nonlocal field strength operators and gauge links bridging the nonlocality. We parametrize the leading (twist-2) gluon-gluon correlator in terms of transverse momentum dependent distribution functions for unpolarized, vector and tensor polarized targets (the latter being relevant for spin-1 targets). For a tensor polarized target there are eleven functions among which two are time reversal odd. We discuss bounds on some functions which might become useful for future applications.
\end{abstract}

\section{Introduction}

The cross section of high energy processes can be written as a convolution of hadronic matrix elements and partonic hard cross section, so long as the validity of factorization theorems is assumed. Nonlocal forward matrix elements containing quarks and gluon fields are studied in terms of Parton distribution functions (PDFs). Extending the non locality to the transverse directions offers possibilities to study transverse momentum depended (TMD) PDFs, or simply TMDs. The hadronic matrix element containing two gluon fields was parametrized in terms of TMD PDFs for the first time in [1] and later on proposed in a different form in [2]. These works describe unpolarized and vector polarized targets, relevant for spin- 0 and spin- $\frac{1}{2}$ particles. Recently in [3] the parametrization of the gluon-gluon correlator has been extended to tensor polarization, allowing in fact the inclusion of spin-1 hadrons in the description. The outline of this work is as follows: in Sect. 2 we show the parametrization of the gluon-gluon correlator in terms of gluon TMDs for hadrons with spin up to 1 and in Sect. 3 we present some bounds on the distributions.

\section{Parametrization of the TMD Gluon-Gluon Correlation Function for Hadrons of Spin $\leq 1$}

The correlation function for gluons in hadrons is defined as:

$$
\Gamma^{\left[U, U^{\prime}\right] \mu \nu ; \rho \sigma}(k, P, n, \ldots) \equiv \int \frac{d^{4} \xi}{(2 \pi)^{4}} e^{i k \cdot \xi}\left\langle P, \ldots\left|F^{\mu \nu}(0) U_{[0, \xi]} F^{\rho \sigma}(\xi) U_{[\xi, 0]}^{\prime}\right| P, \ldots\right\rangle,
$$

This article belongs to the Topical Collection "Light Cone 2016".

S. Cotogno $(\bowtie)$

Nikhef, Science Park 105, 1098 XG Amsterdam, The Netherlands

E-mail: scotogno@ nikhef.nl

S. Cotogno

Department of Physics and Astronomy, VU University Amsterdam, De Boelelaan 1081, 1081 HV Amsterdam, The Netherlands 
where a trace in color space $\left(\operatorname{Tr}_{c}\right)$ is implicitly assumed. The Wilson lines $U_{[0, \xi]}$ and $U_{[\xi, 0]}^{\prime}$ guarantee color gauge invariance as they bridge the nonlocality of the two gluon field tensors. The dots indicate the spin vectors and/or tensors needed to deal with polarization of hadrons with spin $\leq 1$.

The relevant momentum variables are $P$ and $k$, i.e. the hadron and parton momentum respectively. We use the lightlike vector $n$, i.e. $n^{2}=0$, and we parametrize $P$ and $k$ such that they satisfy:

$$
P^{2}=M^{2}, \quad P \cdot n=1, \quad k^{\mu}=x P^{\mu}+k_{T}^{\mu}+\left(k \cdot P-x M^{2}\right) n^{\mu} .
$$

where $M$ is the mass of the hadron.

The target spin states are characterized by the density matrix. For hadrons of spin 0 and $\frac{1}{2}$, one needs to introduce a spacelike vector $S^{\mu}$ which parametrizes the density matrix [4,5]. By demanding $S^{2}=-1$ and $P \cdot S=0$ one can parametrize $S$ in terms of the dimensionful vectors $P$ and $n$ :

$$
S^{\mu}=S_{L} \frac{P^{\mu}}{M}+S_{T}^{\mu}-M S_{L} n^{\mu}
$$

To extend the description to spin-1 particles a polarization tensor $T^{\mu \nu}$ is needed, which satisfies $P_{\mu} T^{\mu \nu}=0$. The parametrization of the tensorial part reads:

$$
\begin{aligned}
T^{\mu \nu}= & \frac{1}{2}\left[\frac{2}{3} S_{L L} g_{T}^{\mu \nu}+\frac{4}{3} S_{L L} \frac{P^{\mu} P^{\nu}}{M^{2}}+\frac{S_{L T}^{\{\mu} P^{\nu\}}}{M}+S_{T T}^{\mu \nu}\right. \\
& \left.-\frac{4}{3} S_{L L} P^{\{\mu} n^{\nu\}}-M S_{L T}^{\{\mu} n^{\nu\}}+\frac{4}{3} M^{2} S_{L L} n^{\mu} n^{\nu}\right] .
\end{aligned}
$$

We have used the metric tensor in transverse space defined as $g_{T}^{\mu \nu} \equiv g^{\mu \nu}-P^{\{\mu} n^{\nu\}}$ (curly brackets denote symmetrization of the indices), with nonvanishing elements $g_{T}^{11}=g_{T}^{22}=-1$. The spin tensor is symmetric and traceless and it has five independent parameters, namely $S_{L L}$, the two components of the transverse vector $S_{L T}$, and the two independent components of the symmetric traceless transverse tensor $S_{T T}$.

Looking at Eq. (1) we note that, even though for unspecified processes the path could run along arbitrary paths, we have included a dependence on the lightlike four-vector $n$, that enters upon consideration of staplelike gauge links running along the light-front $(\xi \cdot n=0)$ via lightlike $\xi \cdot P= \pm \infty$. Integrating Eq. (1) over $k \cdot P$, one obtains the TMD (light-front) correlator

$$
\left.\Gamma^{\left[U, U^{\prime}\right] \mu \nu ; \rho \sigma}\left(x, \boldsymbol{k}_{T} ; P, n\right) \equiv \int \frac{d \xi \cdot P d^{2} \xi_{T}}{(2 \pi)^{3}} e^{i k \cdot \xi}\left\langle P\left|F^{\mu v}(0) U_{[0, \xi]} F^{\rho \sigma}(\xi) U_{[\xi, 0]}^{\prime}\right| P\right\rangle\right|_{\xi \cdot n=0} .
$$

Simple power counting leads to the definition of the leading (usually referred to as leading twist or twist-2) correlator:

$$
\Gamma^{i j}\left(x, \boldsymbol{k}_{T}\right) \equiv \Gamma^{\left[U, U^{\prime}\right] n i ; n j}\left(x, \boldsymbol{k}_{T} ; P, n\right) .
$$

The latter is indeed the operator considered as a starting point in [3], and we repeat the main results on the parametrization therein derived.

In the decomposition we employ symmetric and traceless tensors $k_{T}^{i_{1} \ldots i_{n}}$ that are built from the partonic momentum $k_{T}$. Up to rank $n=4$, these are given by

$$
\begin{aligned}
k_{T}^{i j} \equiv & k_{T}^{i} k_{T}^{j}+\frac{1}{2} \boldsymbol{k}_{T}^{2} g_{T}^{i j}, \\
k_{T}^{i j k} \equiv & k_{T}^{i} k_{T}^{j} k_{T}^{k}+\frac{1}{4} \boldsymbol{k}_{T}^{2}\left(g_{T}^{i j} k_{T}^{k}+g_{T}^{i k} k_{T}^{j}+g_{T}^{j k} k_{T}^{i}\right), \\
k_{T}^{i j k l} \equiv & k_{T}^{i} k_{T}^{j} k_{T}^{k} k_{T}^{l}+\frac{1}{6} \boldsymbol{k}_{T}^{2}\left(g_{T}^{i j} k_{T}^{k l}+g_{T}^{i k} k_{T}^{j l}+g_{T}^{i l} k_{T}^{j k}+g_{T}^{j k} k_{T}^{i l}+g_{T}^{j l} k_{T}^{i k}+g_{T}^{k l} k_{T}^{i j}\right) \\
& -\frac{1}{8} \boldsymbol{k}_{T}^{4}\left(g_{T}^{i j} g_{T}^{k l}+g_{T}^{i k} g_{T}^{j l}+g_{T}^{i l} g_{T}^{j k}\right),
\end{aligned}
$$

satisfying

$$
g_{T i j} k_{T}^{i j}=g_{T i j} k_{T}^{i j k}=g_{T i j} k_{T}^{i j k l}=0
$$


From this decomposition of the Lorentz structures it follows that the functions involved in the parametrization are twist-2 TMDs of definite rank. This has the advantage that there is a one-to-one correspondence between the functions in the transverse coordinate $\boldsymbol{b}_{T}$ space and the $\boldsymbol{k}_{T}$ space, which turns out to be a very important feature when the TMD evolution equations have to be considered [6].

Separation into different polarizations gives:

$$
\Gamma=\Gamma_{U}+\Gamma_{L}+\Gamma_{T}+\Gamma_{L L}+\Gamma_{L T}+\Gamma_{T T}
$$

where:

$$
\begin{aligned}
& \Gamma_{U}^{i j}\left(x, \boldsymbol{k}_{T}\right)=\frac{x}{2}\left[-g_{T}^{i j} f_{1}\left(x, \boldsymbol{k}_{T}^{2}\right)+\frac{k_{T}^{i j}}{M^{2}} h_{1}^{\perp}\left(x, \boldsymbol{k}_{T}^{2}\right)\right], \\
& \Gamma_{L}^{i j}\left(x, \boldsymbol{k}_{T}\right)=\frac{x}{2}\left[i \epsilon_{T}^{i j} S_{L} g_{1}\left(x, \boldsymbol{k}_{T}^{2}\right)+\frac{\epsilon_{T \alpha}^{\{i} k_{T}^{j\} \alpha} S_{L}}{2 M^{2}} h_{1 L}^{\perp}\left(x, \boldsymbol{k}_{T}^{2}\right)\right], \\
& \Gamma_{T}^{i j}\left(x, \boldsymbol{k}_{T}\right)=\frac{x}{2}\left[-\frac{g_{T}^{i j} \epsilon_{T}^{S_{T} k_{T}}}{M} f_{1 T}^{\perp}\left(x, \boldsymbol{k}_{T}^{2}\right)+\frac{i \epsilon_{T}^{i j} \boldsymbol{k}_{T} \cdot \boldsymbol{S}_{T}}{M} g_{1 T}\left(x, \boldsymbol{k}_{T}^{2}\right)\right. \\
& \left.-\frac{\epsilon_{T}^{k_{T}\{i} S_{T}^{j\}}+\epsilon_{T}^{S_{T}\{i} k_{T}^{j\}}}{4 M} h_{1}\left(x, \boldsymbol{k}_{T}^{2}\right)-\frac{\epsilon_{T \alpha}^{\{i} k_{T}^{j\} \alpha S_{T}}}{2 M^{3}} h_{1 T}^{\perp}\left(x, \boldsymbol{k}_{T}^{2}\right)\right], \\
& \Gamma_{L L}^{i j}\left(x, \boldsymbol{k}_{T}\right)=\frac{x}{2}\left[-g_{T}^{i j} S_{L L} f_{1 L L}\left(x, \boldsymbol{k}_{T}^{2}\right)+\frac{k_{T}^{i j} S_{L L}}{M^{2}} h_{1 L L}^{\perp}\left(x, \boldsymbol{k}_{T}^{2}\right)\right], \\
& \Gamma_{L T}^{i j}\left(x, \boldsymbol{k}_{T}\right)=\frac{x}{2}\left[-\frac{g_{T}^{i j} \boldsymbol{k}_{T} \cdot \boldsymbol{S}_{L T}}{M} f_{1 L T}\left(x, \boldsymbol{k}_{T}^{2}\right)+\frac{i \epsilon_{T}^{i j} \epsilon_{T}^{S_{L T} k_{T}}}{M} g_{1 L T}\left(x, \boldsymbol{k}_{T}^{2}\right)\right. \\
& \left.+\frac{S_{L T}^{\{i} k_{T}^{j\}}}{M} h_{1 L T}\left(x, \boldsymbol{k}_{T}^{2}\right)+\frac{k_{T}^{i j \alpha} S_{L T \alpha}}{M^{3}} h_{1 L T}^{\perp}\left(x, \boldsymbol{k}_{T}^{2}\right)\right] \text {, } \\
& \Gamma_{T T}^{i j}\left(x, \boldsymbol{k}_{T}\right)=\frac{x}{2}\left[-\frac{g_{T}^{i j} k_{T}^{\alpha \beta} S_{T T \alpha \beta}}{M^{2}} f_{1 T T}\left(x, \boldsymbol{k}_{T}^{2}\right)+\frac{i \epsilon_{T}^{i j} \epsilon_{T \gamma}^{\beta} k_{T}^{\gamma \alpha} S_{T T \alpha \beta}}{M^{2}} g_{1 T T}\left(x, \boldsymbol{k}_{T}^{2}\right)\right. \\
& +S_{T T}^{i j} h_{1 T T}\left(x, \boldsymbol{k}_{T}^{2}\right)+\frac{S_{T T \alpha}^{\{i} k_{T}^{j\} \alpha}}{M^{2}} h_{1 T T}^{\perp}\left(x, \boldsymbol{k}_{T}^{2}\right) \\
& \left.+\frac{k_{T}^{i j \alpha \beta} S_{T T \alpha \beta}}{M^{4}} h_{1 T T}^{\perp \perp}\left(x, \boldsymbol{k}_{T}^{2}\right)\right] \text {. }
\end{aligned}
$$

Throughout the paper, the dependence of functions on the gauge link is understood and we define $\boldsymbol{k}_{T} \cdot \boldsymbol{S}_{T}=$ $-k_{T} \cdot S_{T}$. The transverse antisymmetric tensor is defined as $\epsilon_{T}^{\mu \nu} \equiv \epsilon^{P n \mu \nu}$, with nonzero components $\epsilon_{T}^{12}=$ $-\epsilon_{T}^{21}=1$. The functions $h_{1 L}^{\perp}, f_{1 T}^{\perp}, h_{1}$, and $h_{1 T}^{\perp}$ are odd under naive time reversal transformatios ( $T$-odd). The functions $h_{1 L L}^{\perp}, f_{1 L T}, h_{1 L T}, h_{1 L T}^{\perp}, f_{1 T T}, h_{1 T T}^{\perp}, h_{1 T T}^{\perp \perp}$ are T-even whereas $g_{1 L T}$ and $g_{1 T T}$ are $T$-odd and they have been first introduced in [3]. The function $f_{1 L L}$ was also called $b_{1}$ in the quark case [7], and $h_{1 T T}$ shows up in the structure function $\Delta\left(x, Q^{2}\right)$ discussed in Ref. [8] and is called $\Delta_{2} G(x)$ in [9]. They are rank-0 functions which survive in the collinear case, i.e. upon integration over transverse momenta.

\section{Positivity Bounds on the Gluon Distributions in Spin-1 Targets}

As already done in [10] one can impose positivity constraints to the hadronic tensor and find a probabilistic interpretation for some of the distribution functions. Bounds on the TMDs have been studied in [1] for gluons in spin- $\frac{1}{2}$ hadrons and in $[7,11]$ for quarks in hadrons with spin up to 1 .

The correlator $\Gamma^{i j}$ in (6) is a $2 \times 2$ matrix in the transverse gluon polarizations. On the other hand, the spin states of a spin-1 particle are described by the density matrix, which can be written in terms of the independent 
components of the vector $S$ and the tensor $T$. In fact one needs a Cartesian basis of $3 \times 3$ matrices, consisting of the identity matrix $I$, the three-dimensional (generalized) Pauli matrices $\Sigma^{i}$, and the five bilinear combinations $\Sigma^{i j} \equiv \frac{1}{2}\left(\Sigma^{i} \Sigma^{j}+\Sigma^{j} \Sigma^{i}\right)-\frac{2}{3} I \delta^{i j}$. The density matrix (in the rest frame) takes the form:

$$
\begin{aligned}
\rho= & \frac{1}{3}\left(I+\frac{3}{2} S^{i} \Sigma^{i}+3 T^{i j} \Sigma^{i j}\right) \\
\rho= & \left(\begin{array}{ccc}
\frac{S_{L}}{2}+\frac{S_{L L}}{3}+\frac{1}{3} & \frac{S_{L T x}-i S_{L T y}}{2 \sqrt{2}}+\frac{S_{T x}-i S_{T y}}{2 \sqrt{2}} & \frac{1}{2}\left(S_{T T x x}-i S_{T T x y}\right) \\
\frac{S_{L T x}+i S_{L T y}}{2 \sqrt{2}}+\frac{S_{T x}+i S_{T y}}{2 \sqrt{2}} & \frac{1}{3}-\frac{2 S L L}{3} & \frac{-S_{L T x}+i S_{L T y}}{2 \sqrt{2}}+\frac{S_{T x}-i S_{T y}}{2 \sqrt{2}} \\
\frac{1}{2}\left(S_{T T x x}+i S_{T T x y}\right) & \frac{-S_{L T x}-i S_{L T y}}{2 \sqrt{2}}+\frac{S_{T x}+i S_{T y}}{2 \sqrt{2}} & -\frac{S L}{2}+\frac{S L L}{3}+\frac{1}{3}
\end{array}\right) .
\end{aligned}
$$

This allows one to write the correlator in a form which explicitly singles out the hadron spin, namely:

$$
\Gamma^{i j}\left(x, \boldsymbol{k}_{\perp} ; P, S, T\right)=\sum_{s, s^{\prime}} \rho_{s^{\prime}, s}(S, T) \Gamma_{s, s^{\prime}}^{i j}\left(x, \boldsymbol{k}_{\perp} ; P\right)
$$

The matrix $\Gamma_{s, s^{\prime}}^{i j}$ in the right hand of (20) is a $6 \times 6$ matrix in the gluon $\otimes$ hadron spin space:

$$
\Gamma_{s, s^{\prime}}^{i j}=\left(\begin{array}{cc}
A & B \\
B^{\dagger} & C
\end{array}\right)
$$

where $A, B$ and $C$ are $3 \times 3$ blocks whose complete expressions will be given elsewhere. From symmetry considerations, it follows that the block $C$ is the charge-conjugated of the block $A$ and the off-diagonal block are hermitian-conjugate (see Ref. [3] for more details on the hermiticity, time-reversal and charge-conjugation properties of the gluon correlator). We give here the explicit form of the matrix (21) in the collinear case where the blocks are:

$$
\begin{aligned}
A & =\left(\begin{array}{ccc}
f_{1}+\frac{f_{1 L L}}{2}-g_{1} & 0 & 0 \\
0 & f_{1}-f_{1 L L} & 0 \\
0 & 0 & f_{1}+\frac{f_{1 L L}}{2}+g_{1}
\end{array}\right), \quad B=\left(\begin{array}{ccc}
0 & 0 & 0 \\
0 & 0 & 0 \\
-2 h_{1 T T} & 0 & 0
\end{array}\right) \\
C & =\left(\begin{array}{ccc}
f_{1}+\frac{f_{1 L L}}{2}+g_{1} & 0 & 0 \\
0 & f_{1}-f_{1 L L} & 0 \\
0 & 0 & f_{1}+\frac{f_{1 L L}}{2}-g_{1}
\end{array}\right)
\end{aligned}
$$

We note that $\Gamma_{s, s^{\prime}}^{i j}$ is a positive semi-definite matrix, i.e. for each vector in the combined gluon $\otimes$ target spin space it satisfies $v^{\dagger} \Gamma v \geq 0$.

$$
\begin{aligned}
\Gamma_{s, s^{\prime}}^{i j}(x ; P) & =\left.\int \frac{d \xi \cdot P}{2 \pi} e^{i k \cdot \xi}\left\langle P, S, T\left|F^{n i}(0) F^{n j}(\xi)\right| P, S, T\right\rangle\right|_{\xi \cdot n=\xi_{T}=0} \\
& =\sum_{m}\left\langle P_{m}\left|F^{n i}(0)\right| P, s\right\rangle^{*}\left\langle P_{m}\left|F^{n j}(0)\right| P, s^{\prime}\right\rangle \delta\left(P_{m} \cdot n-(1-x) P \cdot n\right)
\end{aligned}
$$

This is true for the unintegrated version of $\Gamma$ as well (see [1,5]). The matrix (21) is positive semi-definite if its eigenvalues are all $\geq 0$. Together with the requirement for all the diagonal elements to be positive, we find the following bounds for the integrated gluon distribution functions:

$$
\begin{aligned}
& f_{1} \geq f_{1 L L}, \\
& \left|g_{1}\right| \leq f_{1}+\frac{f_{1 L L}}{2} \leq \frac{3}{2} f_{1} \\
& \left|h_{1 T T}\right| \leq \frac{1}{2}\left(f_{1}+\frac{f_{1 L L}}{2}+g_{1}\right) .
\end{aligned}
$$

Including transverse momenta produces many more relations on the functions which will be shown in a future work. 


\section{Conclusions}

We have presented the parametrization of the gluon-gluon correlator in terms of TMDs and we have considered targets with spin up to 1 . Some of the new functions presented in [3] survive under integration over transverse momenta and we derived the bounds fullfilled by these collinear distributions.

Acknowledgements This work was done in collaboration with D. Boer, T. van Daal, P. J. Mulders, A. Signori and Y. Zhou. The research was financially supported by the European Research Council (ERC) under the program QWORK (Contract No. 320389) and the "Nederlandse Organisatie voor Wetenschappelijk Onderzoek (NWO)".

Open Access This article is distributed under the terms of the Creative Commons Attribution 4.0 International License (http:// creativecommons.org/licenses/by/4.0/), which permits unrestricted use, distribution, and reproduction in any medium, provided you give appropriate credit to the original author(s) and the source, provide a link to the Creative Commons license, and indicate if changes were made.

\section{References}

1. P.J. Mulders, J. Rodrigues, Transverse momentum dependence in gluon distribution and fragmentation functions. Phys. Rev. D 63, 094021 (2001). [arXiv:hep-ph/0009343]

2. S. Meissner, A. Metz, K. Goeke, Relations between generalized and transverse momentum dependent parton distributions. Phys. Rev. D 76, 034002 (2007). [arXiv:hep-ph/0703176 [HEP-PH]]

3. D. Boer, S. Cotogno, T. van Daal, P.J. Mulders, A. Signori, Y.J. Zhou, Gluon and Wilson loop TMDs for hadrons of spin $\leq$ 1. JHEP 1610, 013 (2016). [arXiv:1607.01654 [hep-ph]]

4. E. Leader, Spin in particle physics. Camb. Monogr. Part. Phys. Nucl. Phys. Cosmol. 15, 1 (2011)

5. A. Bacchetta, P.J. Mulders, Deep inelastic leptoproduction of spin-one hadrons. Phys. Rev. D 62, 114004 (2000). [arXiv:hep-ph/0007120]

6. A. Signori, Flavor and evolution effects in TMD phenomenology: manifestation of Hadron structure in high-energy scattering processes (2016), https://inspirehep.net/record/1493030/files/Thesis-2016-Signori.pdf. Accessed 14 Feb 2017

7. A. Bacchetta, Probing the transverse spin of quarks in deep inelastic scattering. arXiv:hep-ph/0212025

8. R.L. Jaffe, A. Manohar, Nuclear gluonometry. Phys. Lett. B 223, 218 (1989)

9. X. Artru, M. Mekhfi, Transversely polarized parton densities, their evolution and their measurement. Z. Phys. C 45, 669 (1990)

10. W. Lu, J.J. Yang, Bounding the Levelt-Mulders asymmetry from the positivity of the hadronic tensor and Callan-Gross relation. Z. Phys. C 73, 689 (1997). [arXiv:hep-ph/9601255]

11. A. Bacchetta, M. Boglione, A. Henneman, P.J. Mulders, Bounds on transverse momentum dependent distribution and fragmentation functions. Phys. Rev. Lett. 85, 712 (2000). [arXiv:hep-ph/9912490] 